\title{
Normal values and within-subject variability of cardiac I-123 MIBG scintigraphy in healthy individuals: Implications for clinical studies
}

\author{
G. Aernout Somsen, MD, PhD, ${ }^{\text {a }}$ Hein J. Verberne, MD, ${ }^{\mathrm{b}}$ Eric Fleury, ${ }^{\mathrm{a}}$ and Alberto \\ Righetti, $M D^{a}$
}

Background. Although several myocardial iodine 123 metaiodobenzylguanidine (MIBG) indices are increasingly used to detect alterations in myocardial sympathetic activity in various forms of cardiac pathology, published measurements of normal values and within-subject variability are lacking.

Methods and Results. Twenty-five healthy volunteers underwent planar and single photon emission computed tomography (SPECT) imaging. Heart-mediastinum ratio (H/M) and myocardial washout were calculated from planar images comparing three different methods for the assessment of myocardial activity: (1) global region over the myocardium (cavity included), (2) global region over the myocardium (cavity excluded), and (3) fixed small myocardial region. Segmental (relative) uptake and washout were assessed by SPECT. For all MIBG indices, the interindividual variation was the lowest for methods 1 and 2. In SPECT this variation was low for relative segmental uptake compared with washout. In 9 subjects a second MIBG scintigraphy was performed after 3 months. The within-subject variability of $\mathbf{H} / \mathrm{M}$ and washout assessed by planar methods 1 and 2 was 5\%, whereas it was approximately $9 \%$ for planar method 3. For relative segmental uptake from SPECT, this variability was $5 \%$.

Conclusion. MIBG H/M (planar) and relative segmental uptake (SPECT) show a low interindividual and within-subject variability. This enables the detection of small (regional) variations in myocardial sympathetic nervous function, especially to monitor the effect of therapeutic interventions in patients with various cardiac diseases. ( $J$ Nucl Cardiol 2004;11: 126-33.)

Key Words: Iodine 123 metaiodobenzylguanidine $\bullet$ scintigraphy $\bullet$ single photon emission computed tomography $\bullet$ normal subjects

In patients with various cardiac diseases increased sympathetic neuronal activity has been related to disease severity and prognosis. ${ }^{1,2}$ Cardiac sympathetic neuronal function and activity can noninvasively be assessed by use of iodine 123-radiolabeled metaiodobenzylguanidine (MIBG), an analog of noradrenaline. As reduced cardiac I-123 MIBG uptake has been related to prognosis, this radiotracer can be used to evaluate the effect of therapeutic interventions on cardiac sympathetic activity. Angiotensin-converting enzyme inhibitors, ${ }^{3,4} \beta$-receptor

From the Department of Cardiology, University Hospital Geneva, Geneva, Switzerland, ${ }^{\mathrm{a}}$ and Department of Nuclear Medicine, ${ }^{\mathrm{b}}$ Academic Medical Center, Amsterdam, The Netherlands.

Received for publication July 21, 2003; final revision accepted Oct 28, 2003.

Reprint requests: G. Aernout Somsen, MD, PhD, Centre de Cardiologie, Hôpital Universitaire de Genève, 24 rue Micheli-du-Crest, 1211 Genève 14, Switzerland; aerout.somsen@HCUGE.CH.

Copyright $\odot 2004$ by the American Society of Nuclear Cardiology. $1071-3581 / 2004 / \$ 30.00+0$

doi:10.1016/j.nuclcard.2002.10.010 antagonists, ${ }^{5-8}$ and spironolactone, ${ }^{9,10}$ which have been shown to ameliorate functional capacity and prognosis in patients with chronic heart failure, have been shown to increase cardiac I-123 MIBG uptake and reduce its washout in these patients, indicating favorable effects on the sympathetic nervous system. Therefore cardiac I-123 MIBG imaging has become a widely used, valuable noninvasive tool for the assessment of prognosis and therapeutic effects in various forms of cardiac pathology.

A drawback of the majority of the previous studies is their nonrandomized and open-label design, because of the small number of patients studied and the necessity for close clinical monitoring during dose titration of study medication. As patients often serve as their own reference in these studies, normal values and the physiological variation of I-123 MIBG indices within the same patient over time have to be assessed. In particular, this within-subject variability needs to be evaluated to interpret the significance of the detected alterations in I-123 MIBG uptake and washout resulting from therapeutic interventions. 
Table 1. Clinical characteristics of healthy volunteers $(n=25)$

\begin{tabular}{|c|c|c|c|c|}
\hline & \multicolumn{2}{|c|}{ Single study $(n=25)$} & \multicolumn{2}{|c|}{ Repeated study $(\mathrm{n}=9)$} \\
\hline & Mean \pm SD & Range & Mean \pm SD & Range \\
\hline Age $(y)$ & $30.5 \pm 8.5$ & $23-56$ & $29.6 \pm 10.5$ & $24-56$ \\
\hline Men/women & $13 / 12$ & & $4 / 5$ & \\
\hline Weight (kg) & $63.4 \pm 9.6$ & $48-85$ & $61.9 \pm 9.9$ & $49-80$ \\
\hline Length $(\mathrm{cm})$ & $171.6 \pm 7.9$ & $159-190$ & $170 \pm 6.7$ & $159-178$ \\
\hline $\mathrm{SBP}(\mathrm{mm} \mathrm{Hg})$ & $124 \pm 8.7$ & $105-140$ & $123 \pm 10.9$ & $110-140$ \\
\hline DBP (mm Hg) & $79 \pm 6.7$ & $70-90$ & $79.4 \pm 5.3$ & $70-90$ \\
\hline HR (beat/min) & $65 \pm 11$ & $45-90$ & $70 \pm 12$ & $61-90$ \\
\hline
\end{tabular}

$S B P$, Systolic blood pressure; $D B P$, diastolic blood pressure; $H R$, heart rate.

To assess normal values and the within-subject variability of commonly used I-123 MIBG indices, we performed cardiac I-123 MIBG scintigraphy in a relatively large group of healthy subjects.

\section{METHODS}

\section{Study Population}

Healthy volunteers without a history of cardiac disease and with a less than 5\% likelihood for coronary artery disease, with age and known risk factors taken into account, were included in the study. Subjects were not taking any medication. To assess within-subject variability, patients were studied twice at a time interval of 3 months. The characteristics of the subjects included in the study are shown in Table 1. The Medical Ethics Committee of our institution approved the study. Written informed consent was obtained from all subjects enrolled in the study.

\section{Acquisition Protocol}

Thyroid uptake was blocked by use of $200 \mathrm{mg}$ potassium iodide 1 hour before injection of $148 \mathrm{MBq}$ I-123 MIBG (radiochemical purity, $\geq 97 \%$; specific activity, $148 \mathrm{MBq}$ I-123/mg MIBG) (Mallinckrodt Schweiz AG, Zürich, Switzerland). MIBG scintigraphy was performed with a dual-head camera (ADAC Epicardio, Milpitas, Calif) equipped with a VXHR collimator. At 15 minutes (early) and 240 minutes (late) after injection of the radiopharmaceutical, planar images of the thorax were obtained during a 10-minute period in both the left anterior oblique (LAO) and anterior projections. Subsequently, tomographic images were obtained with the use of 32 frames over $180^{\circ}\left(90^{\circ}\right.$ per camera head) for 60 seconds per frame at 35 minutes (early) and 260 minutes (late) after tracer injection. A $20 \%$ energy window was centered on the $159-\mathrm{keV}$ photopeak of I-123. Data collection was performed with a $128 \times 128$ matrix and a zoom factor of 1.46 . The studies were reconstructed with the PEGASYS software package (ADAC Laboratories, Milpitas, Calif) and fifth- order Pega Butterworth prereconstruction filter. Images were zoomed to $250 \%$. No correction for attenuation was applied because, at the time of acquisition, no validated algorithm was available for I-123. Moreover, the majority of previous studies did not use this correction, which allows for better comparison with data from this study. Slice thickness was 1 pixel $(0.64 \mathrm{~cm})$.

\section{Image Analysis}

Planar images. Cardiac MIBG uptake was determined by three clinically used methods (Figure 1). For method 1, a region of interest (ROI) was drawn over the myocardium including the left ventricular cavity. ${ }^{1,11,12}$ For method 2, an ROI was drawn over the myocardium excluding the left ventricular cavity. For method 3, peak count densities for the myocardium and upper mediastinum were determined. ${ }^{4,13}$ For methods 1 and 2, ROIs were set over the upper mediastinum (in the anterior projection with a fixed area of 40 pixels) and manually drawn for the heart (anterior and LAO projection in both). The mean count density (for methods 1 and 2) and the peak count densities (for method 3 ) were calculated and corrected for injected dose and body weight. I-123 MIBG uptake was expressed as the count density ratio between the heart and the upper mediastinum $(\mathrm{H} / \mathrm{M})$. The myocardial I-123 MIBG (decay-corrected) washout was calculated by use of the count densities (CD) according to the following algorithm:

$$
\begin{gathered}
\text { Cardiac I-123 MIBG washout }=\left(\mathrm{CD}_{\text {heart-early }}-\mathrm{CD}_{\text {heart-late }} /\right. \\
\left.\mathrm{CD}_{\text {heart-early }}\right) \times 100 \%
\end{gathered}
$$

Single photon emission computed tomography images. From tomographic images, cardiac short-axis slices were reconstructed from basis to apex for bull's-eye reconstruction. Three myocardial segments were manually drawn (Figure 2), and segmental volumes were reconstructed. Segmental count density and (decay-corrected) washout were calculated and expressed as a percentage of total myocardial count density in tomographic images. 


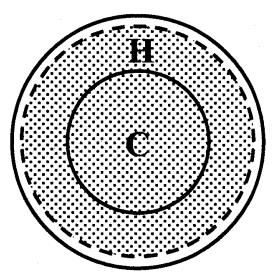

method 1

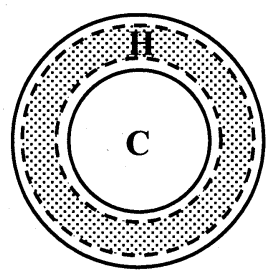

method 2

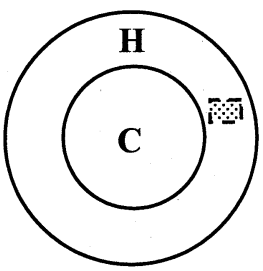

method 3

Figure 1. Schematic view of ROIs (shaded areas) drawn on planar images according to three clinically used quantification methods: an ROI drawn for the myocardium including the left ventricular cavity (method 1), an ROI drawn for the myocardium excluding the left ventricular cavity (method 2), and peak count densities for the myocardium and the upper mediastinum (method 3). $H$, Heart; $C$, cavity. For methods 1 and 2, an ROI of 40 pixels was placed in the mediastinum as a reference.

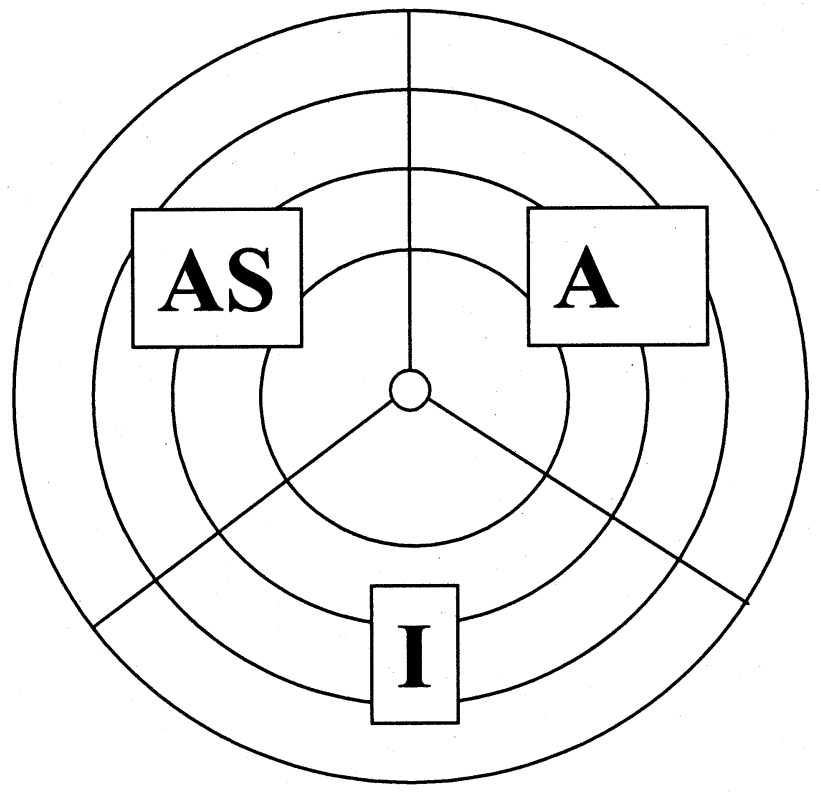

Figure 2. Segmental analysis of I-123 MIBG uptake on SPECT images with a polar map. $A S$, Anteroseptal; $A L$, anterolateral; $I$, inferior.

\section{Statistical Analysis}

All values are expressed as mean $\pm \mathrm{SD}$. Correlations were expressed as Pearson correlation coefficients $(r)$. The withinsubject variability was assessed by use of the coefficient of variation $(\mathrm{CV})$. The within-subject $\mathrm{CV}$ of repeated measurements was calculated as follows (in which $x$ and $y$ represent two observations, at baseline and after 3 months $)^{14}$ :

$$
\begin{aligned}
s & =(x-y)^{2} / 2, \quad m=([x+y] / 2)^{2} \\
\mathrm{CV} & =\text { Square root of (mean } s / \text { mean } m) \\
s & =\text { standard deviation, } m=\text { mean }
\end{aligned}
$$

For each CV, the $95 \%$ confidence interval was calculated. Univariate analysis and forward stepwise regression analysis were performed for the identification of explanatory independent variables of the various MIBG indices. Independent variables tested were weight, age, length, heart rate, and both systolic and diastolic blood pressure at the time of acquisition. A $P$ value $<.05$ was considered to represent statistical significance. For all statistical analyses, StatView 4.57 (Abacus Concepts Inc, Berkeley, Calif) was used.

\section{RESULTS}

Twenty-five healthy volunteers were included in the study and underwent cardiac I-123 MIBG scintigraphy. Nine subjects were studied twice, with a time interval of 3 months, to evaluate within-subject variability. All studies could be reconstructed and analyzed.

\section{Normal Values of I-123 MIBG Indices}

Planar images. For all planar methods used, the interindividual variation, as expressed by the SD, was relatively high for cardiac uptake, whereas for mediastinum uptake, H/M, and cardiac washout, this variation appeared to be lower (Tables 2, 3, and 4). For myocardial uptake, H/M, and washout, the interindividual variation was lower for method 1 compared with methods 2 and 3. The lowest H/M was found for method 1, whereas the highest $\mathrm{H} / \mathrm{M}$ was found for method 3. However, when the three methods were compared, a higher H/M was associated with larger interindividual variation. Interindividual variation for $\mathrm{H} / \mathrm{M}$ in the anterior projection was slightly less than in the LAO projection. There was a trend toward higher $\mathrm{H} / \mathrm{M}$ in the anterior compared with the LAO projection without reaching the level of significance. No gender differences were found for any MIBG index.

Single photon emission computed tomography images. For single photon emission computed tomography (SPECT) acquisitions, a large interindividual vari- 
Table 2. Method 1: I-123 MIBG count densities (corrected for dose and body weight), H/M, and cardiac washout for various projections in planar imaging $(n=25)$

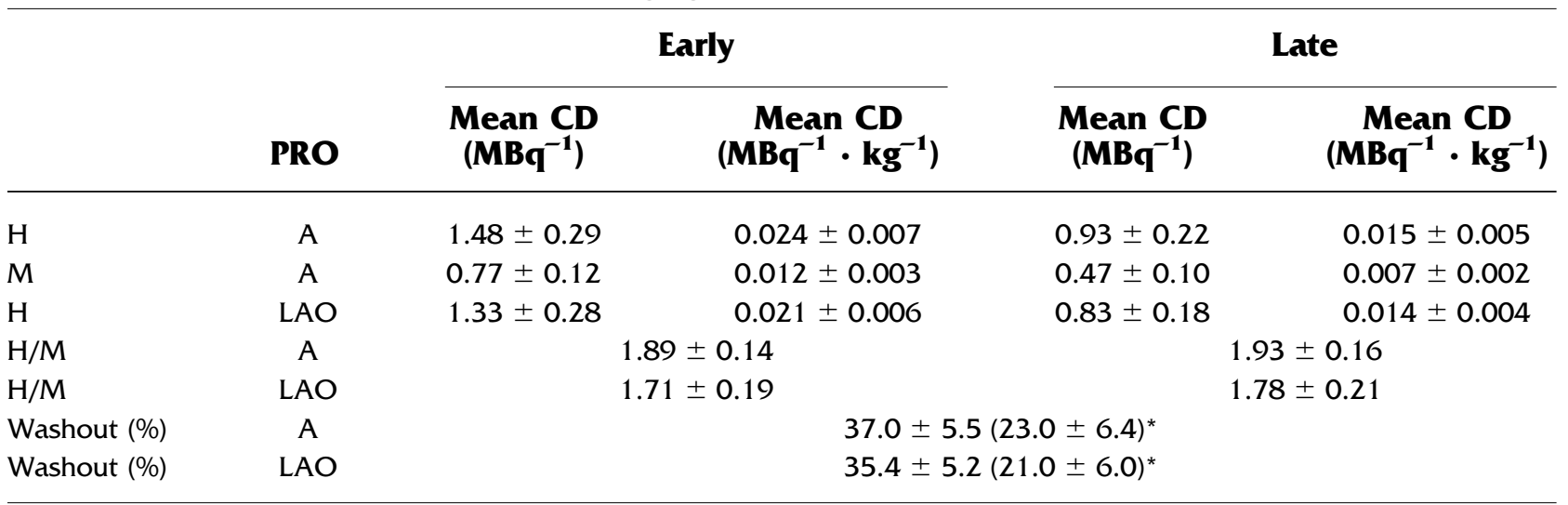

$C D$, count density; $H$, Heart; $M$, mediastinum; PRO, projection used to obtain myocardial ROI; $A$, anterior.

*Decay-corrected values.

Table 3. Method 2: I-123 MIBG count densities (corrected for dose and body weight), H/M, and cardiac washout for various projections in planar imaging $(n=25)$

\begin{tabular}{|c|c|c|c|c|c|}
\hline & \multirow[b]{2}{*}{ PRO } & \multicolumn{2}{|c|}{ Early } & \multicolumn{2}{|c|}{ Late } \\
\hline & & $\begin{array}{c}\text { Mean CD } \\
\left(\mathbf{M B q}^{-1}\right)\end{array}$ & $\begin{array}{c}\text { Mean CD } \\
\left(\mathrm{MBq}^{-1} \cdot \mathrm{kg}^{-1}\right)\end{array}$ & $\begin{array}{c}\text { Mean CD } \\
\left(\mathbf{M B q}^{-1}\right)\end{array}$ & $\begin{array}{c}\text { Mean CD } \\
\left(\mathrm{MBq}^{-1} \cdot \mathbf{k g}^{-1}\right)\end{array}$ \\
\hline $\mathrm{H}$ & A & $1.55 \pm 0.31$ & $0.025 \pm 0.007$ & $0.99 \pm 0.24$ & $0.016 \pm 0.005$ \\
\hline$M$ & A & $0.77 \pm 0.12$ & $0.012 \pm 0.003$ & $0.47 \pm 0.10$ & $0.007 \pm 0.002$ \\
\hline $\mathrm{H}$ & LAO & $1.40 \pm 0.27$ & $0.023 \pm 0.007$ & $0.93 \pm 0.22$ & $0.015 \pm 0.005$ \\
\hline $\mathrm{H} / \mathrm{M}$ & A & \multicolumn{2}{|c|}{$2.02 \pm 0.20$} & \multicolumn{2}{|c|}{$2.10 \pm 0.24$} \\
\hline $\mathrm{H} / \mathrm{M}$ & LAO & \multicolumn{2}{|c|}{$1.83 \pm 0.19$} & \multicolumn{2}{|c|}{$1.98 \pm 0.25$} \\
\hline Washout (\%) & A & \multicolumn{4}{|c|}{$36.0 \pm 6.0(23.7 \pm 6.9)^{*}$} \\
\hline Washout (\%) & LAO & \multicolumn{4}{|c|}{$33.0 \pm 9.0(20.1 \pm 11.3)^{*}$} \\
\hline
\end{tabular}

$C D$, count density; $H$, Heart; $M$, mediastinum; PRO, projection used to obtain myocardial ROI; $A$, anterior.

${ }^{*}$ Decay-corrected values.

ation was found for total myocardial and segmental uptake (expressed as count density) and washout (Table 5). However, when segmental uptake in tomographic images was expressed as percent of maximum myocardial uptake, this variation was reduced. In early and late images, percent I-123 MIBG uptake in the inferior segment was significantly lower than in the lateral segments $(P<.0001)$. No gender differences were found for any MIBG index.

Univariate analysis. Univariate analysis demonstrated no significant correlation between any independent variable and any planar-derived MIBG indices. Weight and length were the only independent explanatory variables for the SPECT-derived myocardial MIBG count densities in early $(r=-0.49, P=.01$, and $r=$ $-0.43, P=.03$, respectively) and late $(r=-0.43, P=$ .03 , and $r=-0.43, P=.04$, respectively) images.
Cardiac washout was not correlated with any independent variable. The weight-corrected count densities for each method are shown in Tables 2, 3, and 4.

Forward stepwise regression analysis. Only weight entered the model as a significant determinant of early cardiac I-123 MIBG uptake (but not late uptake or washout).

\section{Within-Subject Variability}

The clinical characteristics of 9 individuals who were studied twice did not differ from the overall normal population (Table 1). During the follow-up of 3 months, no changes in clinical characteristics were observed. All planar and tomographic data are shown in Tables 6 and 7, respectively. On planar images, $\mathrm{H} / \mathrm{M}$ and cardiac washout were characterized by low within-subject vari- 
Table 4. Method 3: I-123 MIBG count densities (corrected for dose and body weight), H/M, and cardiac washout for various projections in planar imaging $(n=25)$

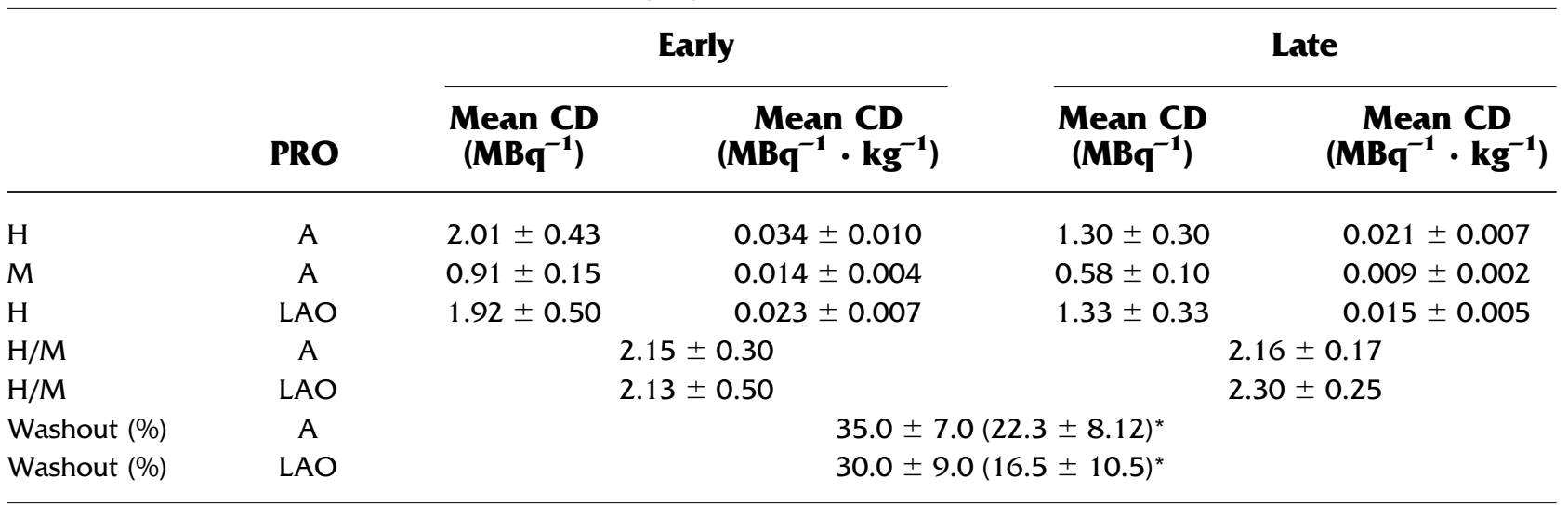

$C D$, count density; $H$, Heart; $M$, mediastinum; PRO, projection used to obtain myocardial ROI; $A$, anterior.

*Decay-corrected values.

Table 5. Segmental I-123 MIBG uptake and washout in tomographic scintigraphy $(\mathrm{n}=25)$

\begin{tabular}{|c|c|c|c|c|c|c|c|}
\hline & \multicolumn{3}{|c|}{ Early } & \multicolumn{3}{|c|}{ Late } & \multirow{2}{*}{$\begin{array}{c}\text { Decay- } \\
\text { corrected } \\
\text { washout } \\
(\%)\end{array}$} \\
\hline & $\begin{array}{c}\text { Mean CD } \\
\left(\mathrm{MBq}^{-1}\right)\end{array}$ & $\begin{array}{c}\text { Mean CD } \\
\left(\mathrm{MBq}^{-1}\right. \\
\left.\mathrm{kg}^{-1}\right)\end{array}$ & $\begin{array}{c}\% \text { of } \\
\text { maximum } \\
\text { uptake }\end{array}$ & $\begin{array}{c}\text { Mean CD } \\
\left(\mathbf{M B q}^{-1}\right)\end{array}$ & $\begin{array}{c}\text { Mean CD } \\
\left(\mathrm{MBq}^{-1}\right. \\
\left.\mathrm{kg}^{-1}\right)\end{array}$ & $\begin{array}{c}\% \text { of } \\
\text { maximum } \\
\text { uptake }\end{array}$ & \\
\hline Heart & $1.01 \pm 0.32$ & $0.016 \pm 0.007$ & - & $0.74 \pm 0.24$ & $0.012 \pm 0.005$ & - & $27.0 \pm 7.411 .4 \pm 8.9$ \\
\hline Septum & $0.96 \pm 0.31$ & $0.016 \pm 0.006$ & $0.98 \pm 0.06$ & $0.72 \pm 0.23$ & $0.012 \pm 0.005$ & $0.95 \pm 0.05^{*}$ & $24.4 \pm 8.0 \quad 8.2 \pm 9.7$ \\
\hline Lateral & $1.11 \pm 0.34$ & $0.018 \pm 0.007$ & $1.07 \pm 0.05$ & $0.79 \pm 0.26$ & $0.013 \pm 0.005$ & $1.10 \pm 0.05$ & $28.8 \pm 8.413 .6 \pm 10.2$ \\
\hline Inferior & $0.97 \pm 0.33$ & $0.016 \pm 0.007$ & $0.95 \pm 0.07^{*}$ & $0.70 \pm 0.23$ & $0.011 \pm 0.005$ & $0.95 \pm 0.07^{*}$ & $27.3 \pm 8.211 .8 \pm 9.9$ \\
\hline
\end{tabular}

${ }^{*} P<.0001$ compared with percent uptake in lateral segments.

ability, especially for methods 1 and 2 by use of the anterior projection. Method 3 was inferior with respect to within-subject variability compared with the other methods, as the CV was the highest for all indices. Between methods 1 and 2, the within-subject variability did not differ for any indices in the anterior projection whereas the within-subject variability was slightly higher in the LAO projection with method 2. Figure 3 illustrates the relationship between the initial measurements and the follow-up measurements for the three different methods.

On tomographic images, a large within-subject variability was shown for segmental count densities and washout (CV ranging from 0.11 to 0.14). However, segmental uptake expressed as a percentage of total myocardial uptake showed a very low within-subject variability (Table 7).

\section{DISCUSSION}

In this study we showed the values of I-123 MIBG indices and the within-subject variability in the largest group of normal individuals ever reported in the literature. The I-123 MIBG parameters commonly used in clinical studies, such as $\mathrm{H} / \mathrm{M}$ and cardiac washout, assessed in planar images, showed a small interindividual variability compared with the dose-corrected count densities in the heart and mediastinum separately.

Although we anticipated an advantage to obtaining the myocardial count densities in the LAO projection (less overprojection of pulmonary tissue), a systematically smaller myocardial ROI resulted in a higher interindividual variability, as a result of unfavorable count statistics, for all three planar methods used. In addition, nonsignificantly lower $\mathrm{H} / \mathrm{M}$ and washout values were found in the LAO projection compared with the anterior projection. Therefore myocardial I-123 MIBG uptake is preferably assessed in the anterior projection to ensure the lowest variation and the highest resolution.

The planar quantification method 3 was characterized by the highest interindividual variability for all indices as compared with the other planar methods. This may be due to the fact that peak count density in the 
Table 6. Within-subject variability (3-month interval) of I-123 MIBG parameters in planar scintigraphy for each $\operatorname{method}(\mathrm{n}=9)$

\begin{tabular}{|c|c|c|c|c|c|c|c|}
\hline \multirow[b]{2}{*}{ Parameter } & \multirow[b]{2}{*}{ PRO } & \multicolumn{2}{|c|}{ Method 1} & \multicolumn{2}{|c|}{ Method 2} & \multicolumn{2}{|c|}{ Method 3} \\
\hline & & $\mathbf{C V}$ & 95\% CI & CV & $95 \% \mathrm{CI}$ & CV & $95 \% \mathrm{CI}$ \\
\hline $\mathrm{H} / \mathrm{M}$ (early) & A & 0.05 & $0.03-0.07$ & 0.05 & $0.03-0.07$ & 0.09 & $0.04-0.13$ \\
\hline $\mathrm{H} / \mathrm{M}$ (late) & A & 0.04 & $0.02-0.05$ & 0.04 & $0.02-0.06$ & 0.06 & $0.02-0.08$ \\
\hline Washout & A & 0.05 & $0.02-0.07$ & 0.06 & $0.02-0.09$ & 0.11 & $0.05-0.15$ \\
\hline Decay-corrected washout & A & 0.09 & $0.00-0.15$ & 0.12 & $0.03-0.17$ & 0.21 & $0.13-0.27$ \\
\hline $\mathrm{H} / \mathrm{M}$ (early) & LAO & 0.06 & $0.04-0.08$ & 0.08 & $0.05-0.10$ & 0.14 & $0.00-0.21$ \\
\hline $\mathrm{H} / \mathrm{M}$ (late) & LAO & 0.07 & $0.02-0.10$ & 0.07 & $0.00-0.10$ & 0.09 & $0.02-0.13$ \\
\hline Washout & LAO & 0.05 & $0.03-0.08$ & 0.08 & $0.00-0.13$ & 0.19 & $0.12-0.26$ \\
\hline Decay-corrected washout & LAO & 0.11 & $0.00-0.17$ & 0.16 & $0.00-0.25$ & 0.82 & $0.00-1.25$ \\
\hline
\end{tabular}

$P R O$, Projection used to obtain myocardial ROI; $95 \% C I$, $95 \%$ confidence interval of $\mathrm{CV}$; $\mathrm{A}$, anterior.

Table 7. Within-subject variability (3-month interval) of relative (percent of maximal cardiac uptake) segmental I-123 MIBG uptake in tomographic scintigraphy $(\mathrm{n}=9)$

\begin{tabular}{lcclll}
\hline & \multicolumn{2}{c}{ Early } & & \multicolumn{2}{c}{ Late } \\
\cline { 2 - 3 } \cline { 6 - 6 } MIBG indices & $\mathbf{C V}$ & $\mathbf{9 5 \%}$ CI & & CV & $\mathbf{9 5 \% ~ C I ~}$ \\
\hline Septum/heart (\%) & 0.03 & $0.02-0.04$ & & 0.02 & $0.01-0.03$ \\
Lateral/heart (\%) & 0.04 & $0.03-0.05$ & & 0.04 & $0.02-0.06$ \\
Inferior/heart (\%) & 0.04 & $0.02-0.06$ & & 0.05 & $0.03-0.08$
\end{tabular}

95\% CI, 95\% Confidence interval of CV.

myocardium was predominantly found in the inferoapical region, which was highly affected by spillover from extracardiac structures, especially the liver. If heterogeneous myocardial I-123 MIBG uptake, which has been reported in various cardiac diseases, is also taken into account, method 3 can be considered to be less suitable for semiquantitative assessment of global cardiac I-123 MIBG uptake.

For quantification method 2, the $\mathrm{H} / \mathrm{M}$ ratios were slightly higher as compared with those obtained with method 1 . However, a larger interindividual variability in the $\mathrm{H} / \mathrm{M}$ ratios for method 2, which is probably caused by the difficulty in exactly defining the endocardial border, may not result in a higher resolution. Therefore, from these data, it can be concluded that planar methods 1 and 2 are equally reliable for the semiquantitative analysis of cardiac I-123 MIBG uptake.

In this study the planar $\mathrm{H} / \mathrm{M}$ values for quantification method 1 in the anterior projection (early, $1.89 \pm$ 0.14 , and late, $1.96 \pm 0.16$ ) were lower than previously reported H/M values in normal individuals. Morozumi et $\mathrm{al},{ }^{15}$ using the same quantification method, demonstrated early and late $\mathrm{H} / \mathrm{M}$ values of $2.80 \pm 0.25$ and $3.02 \pm$ 0.47 , respectively. Differences in the size of the ROI used for the upper mediastinum corrected for differences in matrix size may explain this inconsistency. Morozumi et al used a fixed ROI of 144 pixels with a matrix size of $256 \times 256$, as compared with 40 pixels with a matrix size of $128 \times 128$ in our study. The ROI used by Morozumi et al recalculated to a matrix size of $128 \times$ 128 decreases by a factor of 4 and is therefore smaller in size compared with our study ( 36 pixels vs 40 pixels, respectively). The smaller ROI leads to a more precise estimation of nonspecific mediastinal uptake and is less influenced by nonspecific lung uptake. This leads to a lower mediastinal count density and therefore explains the differences in H/M ratios. However, a smaller ROI also leads to a larger variability, as reflected by a larger $\mathrm{SD}$ for late $\mathrm{H} / \mathrm{M}$. Our data are more in line with those of Merlet et al. ${ }^{16}$ Using method 1, they reported late H/M values of $1.95 \pm 0.31$ (range, 1.75-2.6).

The values for myocardial washout on planar images were in keeping with previously reported values. ${ }^{15}$ On tomographic images, cardiac washout was significantly lower as compared with cardiac washout on planar images. Because pulmonary washout obtained from our data $(49 \% \pm 2.5 \%)$ is significantly higher than cardiac washout, which is in keeping with data from other authors, ${ }^{15}$ overprojection of pulmonary tissue on planar images may account for this difference. Although intersubject variability is high, washout measured on tomographic images may better reflect sympathetic nervous activity at a myocardial level. However, decay-corrected washout showed a higher interindividual and withinsubject variability compared with non-decay-corrected washout from both planar and SPECT acquisitions. Decay correction not only increases presumed activity 

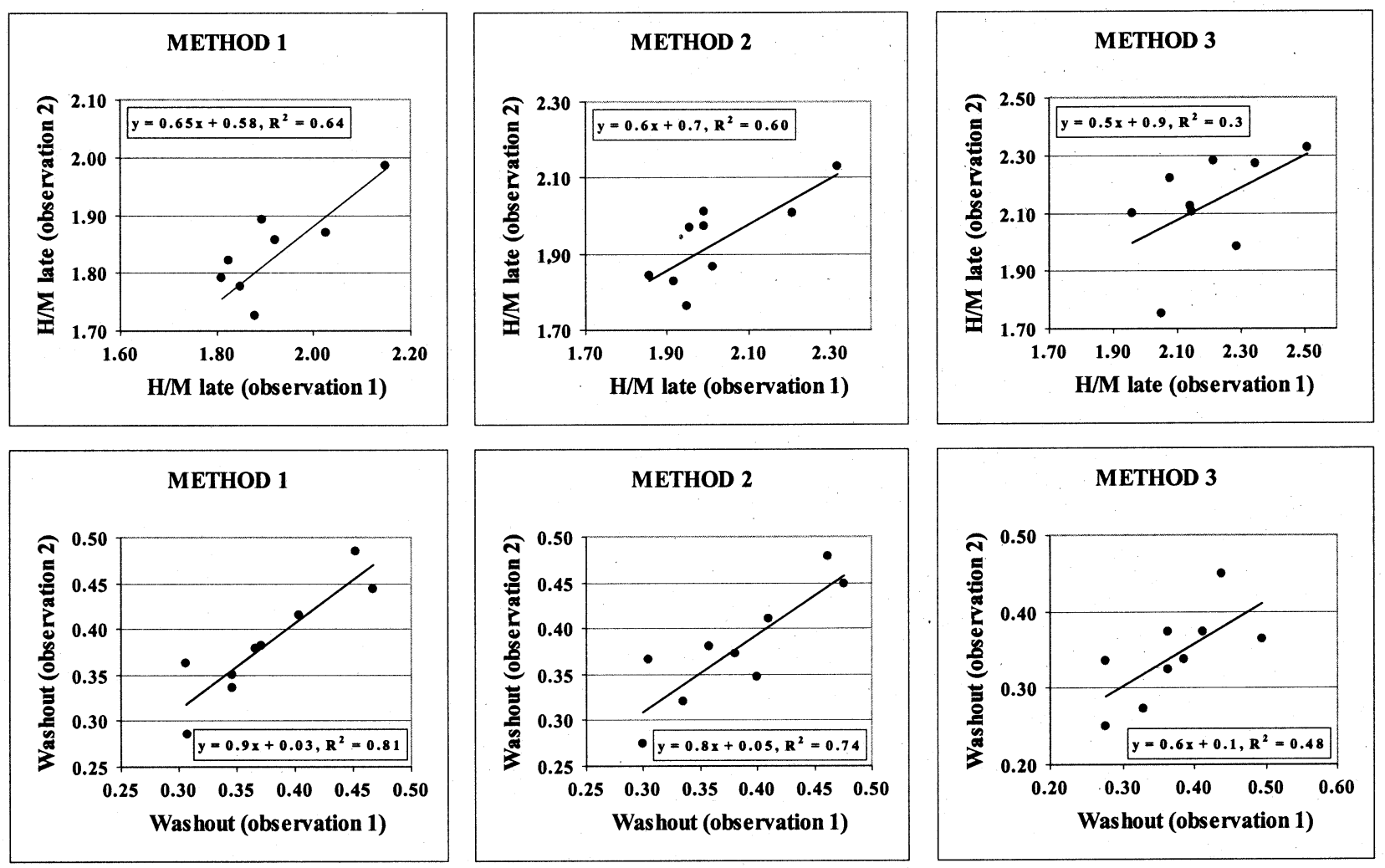

Figure 3. Within-subject variability for each planar method with respect to $\mathrm{H} / \mathrm{M}$ and myocardial washout at baseline (observation 1) and after 3 months (observation 2).

but also increases SD. Therefore decay-corrected myocardial washout showed lower values but higher variation. From a theoretical perspective, correction for physical decay in the calculation of biological washout is understandable. This correction, however, implies an increase in variation. One could therefore wonder whether small changes in sympathetic neuronal activity, as assessed with myocardial I-123 MIBG washout, could be done better without decay correction (ie, smaller variation).

As heterogeneous I-123 MIBG uptake on tomographic images has been shown to be present in normal individuals $^{15,17}$ and patients with heart failure, ${ }^{12}$ we assessed the segmental variability in uptake and washout. Relative I-123 MIBG uptake in the inferior wall was lower compared with the lateral segment, which is in accordance with other studies. ${ }^{15,17}$ This regional difference in I-123 MIBG uptake is probably due to both tissue attenuation and spatial variation in sympathetic nerve activity, as the uptake of carbon 11-epinephrine, which is a positron emission tomography equivalent of I-123 MIBG, was also significantly reduced in the inferior wall in healthy individuals. ${ }^{18}$ The interindividual variation of segmental count densities was large, probably because of low count statistics (typically, 200 counts/pixel). How- ever, when segmental uptake was expressed as a percentage of total myocardial uptake, intersubject variability was low, which supports the use of relative measurements to assess segmental variation in cardiac I-123 MIBG uptake.

Several variables, such as age and body weight, have been shown to be related to cardiac I-123 MIBG uptake and washout. ${ }^{19-22}$ In our study only weight was a consistent independent determinant of various I-123 MIBG SPECT indices in univariate analysis. However, only early cardiac uptake on SPECT images was related to weight in multivariate analysis. Therefore weight correction of the I-123 MIBG SPECT data may be considered for clinical studies. Probably as a result of the smaller age range in our normal population compared with patient data from other studies, age was not an independent determinant of any of the I-123 MIBG myocardial indices.

The within-subject variability was lower for planar than for tomographic acquisitions, which is probably a result of higher count densities on planar images. Planar method 3 was characterized by a high within-subject variability, indicating that this method is not useful for the detection of small alterations in cardiac I-123 MIBG uptake. However, for planar methods 1 and 2 (in the 
anterior projection), a lower CV (approximately 5\%) was demonstrated for all indices. This has important implications for clinical studies, as a $5 \%$ variation over time is low and enables the detection of small alterations in planar I-123 MIBG indices to monitor disease progression or the effect of therapeutic interventions. For tomographic images, within-subject variability was relatively high for segmental count densities. However, when segmental uptake was expressed as a percent of total myocardial uptake, within-subject variability was low, which indicates that relative segmental uptake may serve as a reliable clinical parameter.

\section{Conclusion}

Myocardial I-123 MIBG indices expressed as H/Mand washout-derived planar images, by use of mean count densities, show a low interindividual and withinsubject variability in healthy individuals, which enables the detection of small variations in cardiac sympathetic nervous function. The use of peak count densities to measure I-123 MIBG uptake is less reliable because of high interpatient and within-patient variability. The anterior projection is superior to the LAO projection with respect to variability and resolution of myocardial I-123 MIBG uptake. On tomographic images, segmental I-123 MIBG uptake expressed as a percentage of total myocardial uptake can be used to determine regional variation in sympathetic neuronal function. These findings have important clinical implications for the interpretation of cardiac I-123 MIBG studies, which are commonly used to detect sympathetic neuronal dysfunction and to evaluate the effect of therapeutic intervention on sympathetic nervous function in various forms of cardiac disease.

\section{Acknowledgment}

We thank Inez Foulkes, Joanna Husband, and Bernadette Roy for their excellent technical assistance.

The authors have indicated they have no financial conflicts of interest.

\section{References}

1. Merlet P, Valette H, Dubois-Rande JL, et al. Prognostic value of cardiac metaiodobenzylguanidine imaging in patients with heart failure. J Nucl Med 1992;33:471-7.

2. Ogita H, Shimonagata T, Fukunami M, et al. Prognostic significance of cardiac (123)I metaiodobenzylguanidine imaging for mortality and morbidity in patients with chronic heart failure: a prospective study. Heart 2001;86:656-60.

3. Somsen GA, van Vlies B, de Milliano PA, et al. Increased myocardial [123I]-metaiodobenzylguanidine uptake after enalapril treatment in patients with chronic heart failure. Heart 1996;76:218-22.

4. Takeishi Y, Atsumi H, Fujiwara S, Takahashi K, Tomoike H. ACE inhibition reduces cardiac iodine-123-MIBG release in heart failure. J Nucl Med 1997;38:1085-9.
5. Yamazaki J, Muto H, Kabano T, et al. Evaluation of beta-blocker therapy in patients with dilated cardiomyopathy-clinical meaning of iodine 123-metaiodobenzylguanidine myocardial single-photon emission computed tomography. Am Heart J 2001;141:645-52.

6. Eichhorn EJ, McGhie AL, Bedotto JB, et al. Effects of bucindolol on neurohormonal activation in congestive heart failure. Am J Cardiol 1991;67:67-73.

7. Gerson MC, Craft LL, McGuire N, et al. Carvedilol improves left ventricular function in heart failure patients with idiopathic dilated cardiomyopathy and a wide range of sympathetic nervous system function as measured by iodine 123 metaiodobenzylguanidine. J Nucl Cardiol 2002;9:608-15.

8. de Milliano PA, de Groot AC, Tijssen JG, et al. Beneficial effects of metoprolol on myocardial sympathetic function: Evidence from a randomized, placebo-controlled study in patients with congestive heart failure. Am Heart J 2002;144:E3.

9. Kasama S, Toyama T, Kumakura H, et al. Spironolactone improves cardiac sympathetic nerve activity and symptoms in patients with congestive heart failure. J Nucl Med 2002;43:1279-85.

10. Kasama S, Toyama T, Kumakura H, et al. Effect of spironolactone on cardiac sympathetic nerve activity and left ventricular remodeling in patients with dilated cardiomyopathy. J Am Coll Cardiol 2003;41:574-81.

11. Miyanaga H, Yoneyama S, Kamitani T, et al. Abnormal myocardial uptake and clearance of 123I-labeled metaiodobenzylguanidine in patients with chronic renal failure and autonomic dysfunction. J Nucl Cardiol 1996;3(6 Pt 1):508-15.

12. Parthenakis FI, Prassopoulos VK, Koukouraki SI, et al. Segmental pattern of myocardial sympathetic denervation in idiopathic dilated cardiomyopathy: relationship to regional wall motion and myocardial perfusion abnormalities. J Nucl Cardiol 2002;9:15-22.

13. Sakata K, Shirotani M, Yoshida H, et al. Effects of amlodipine and cilnidipine on cardiac sympathetic nervous system and neurohormonal status in essential hypertension. Hypertension 1999;33:1447-52.

14. Bland JM. Statistics notes: measurement error proportional to the mean [Letter]. BMJ 1996;313:106.

15. Morozumi T, Kusuoka H, Fukuchi K, et al. Myocardial iodine123-metaiodobenzylguanidine images and autonomic nerve activity in normal subjects. J Nucl Med 1997;38:49-52.

16. Merlet P, Benvenuti C, Moyse D, et al. Prognostic value of MIBG imaging in idiopathic dilated cardiomyopathy. J Nucl Med 1999; 40:917-23.

17. Gill JS, Hunter GJ, Gane G, Camm AJ. Heterogeneity of the human myocardial sympathetic innervation: in vivo demonstration by iodine 123-labeled meta-iodobenzylguanidine scintigraphy. Am Heart J 1993;126:390-8.

18. Hubertus Bülow H, Nekolla SG, Schwaiger M, Bengel F. Comparison of the normal distribution of three tracers used for evaluation of the presynaptic sympathetic neuron. J Nucl Cardiol 2003;10:S44.

19. Tsuchimochi S, Tamaki N, Tadamura E, et al. Age and gender differences in normal myocardial adrenergic neuronal function evaluated by iodine-123-MIBG imaging. J Nucl Med 1995;36:969-74.

20. Somsen GA, Borm JJ, Dubois EA, et al. Cardiac 123I-MIBG uptake is affected by variable uptake in reference regions: implications for interpretation in clinical studies. Nucl Med Commun 1996;17:872-6.

21. Estorch M, Carrio I, Berna L, Lopez-Pousa J, Torres G. Myocardial iodine-labeled metaiodobenzylguanidine 123 uptake relates to age. J Nucl Cardiol 1995;2(2 Pt 1):126-32.

22. Leineweber K, Wangemann T, Giessler C, et al. Age-dependent changes of cardiac neuronal noradrenaline reuptake transporter (uptake1) in the human heart. J Am Coll Cardiol 2002;40:145965. 